\title{
Clinical Trial of Lincomycin Hydrochloride in Reiter's Disease
}

\author{
KEITH WHALEY,* M.B., M.R.C.P. ; W. WILSON DOWNIE, $†$ M.B., B.SC. \\ W. CARSON DICK,* M.B., M.R.C.P., D.oBST.R.C.O.G. ; GEORGE NUKI,§ M.B., M.R.C.P. \\ C. B. S. SCHOFIELD, $\|$ M.D., M.R.C.P.ED. ; JOHN ANDERSON, $\mid$ M.A.
}

British Medical fournal, 1969, 2, 421-422

\begin{abstract}
Ummary : A double-blind trial comparing lincomycin hydrochloride (Mycivin) and placebo in 22 patients with Reiter's disease showed no significant difference in clinical or laboratory findings between the two groups. It is concluded that lincomycin hydrochloride is no more effective than placebo in the treatment of Reiter's disease.
\end{abstract}

\section{Introduction}

Reiter's disease is an illness generally characterized by the triad of arthritis, urethritis, and conjunctivitis. It is also associated with a variety of mucocutaneous lesions, such as keratodermia blenorrhagica, circinate balanitis, oral ulceration, and nail changes. It is important to realize, however, that one of the typical features may be absent in any single case. An infective aetiology has been suggested because of its known association with dysentery, and venereal exposure and various infective agents have been implicated. The two which have received the most attention are the mycoplasma and the Bedsonia groups. So far, however, no definite aetiological agent has been identified, and it is of interest that females rarely develop the condition (Rinkoff, 1952). Mowat et al. (1967) presented the case report of a patient with Reiter's disease who responded to lincomycin hydrochloride (Mycivin). After the institution of this therapy there was a fall in the E.S.R., a rise in haemoglobin, and a subsidence of the articular manifestations. On withdrawal of treatment a relapse occurred involving both the local and systemic manifestations. On reintroduction of the therapy a further remission of symptoms occurred.

In view of the possible importance of this observation it was decided to perform a double-blind trial of lincomycin hydrochloride against placebo.

\section{Materials and Methods}

Twenty-two patients with Reiter's disease were included in the study. All were consistently seronegative for rheumatoid factor and had no radiological evidence of bony erosion. Alternate patients were given lincomycin hydrochloride in a dose of $2 \mathrm{~g}$./day or lactose in identical capsules. Treatment was continued for four weeks and the assessment of the clinical findings was made by a physician who was unaware of the nature of the therapy. Each subject was examined clinically before treatment and also twice-weekly throughout the duration of the therapy. Points noted were the articular index, a measurement of joint tenderness (Ritchie et al., 1968), and the presence of urethritis, circinate balanitis, keratodermia blenorrhagica, nail changes, oral ulceration, and conjunctivitis. Further investigation performed at similar times included white cell count and E.S.R. \footnotetext{
* Medical Registrar, Centre for Rheumatic Diseases, Glasgow C.4.
t Arthritis and Rheumatism Lecturer, Royal Infirmary, Glasgow. Ciba Clinical Research Fellow.

Consultant Venereologist, Belvidere Hospital, Glasgow.

$\$$ Department of Biomathematics, University of Oxford.
}

\section{Results}

The clinical and laboratory features of the 22 patients included in the study are shown in Table I. Both groups were closely matched regarding age, duration of disease, articular index, white cell count, and E.S.R. Other clinical features were about as prevalent in either group, apart from conjunctivitis, which was just over twice as common in the placebo-treated group.

TABLE I.-Clinical and Laboratory Features of Patients Included in

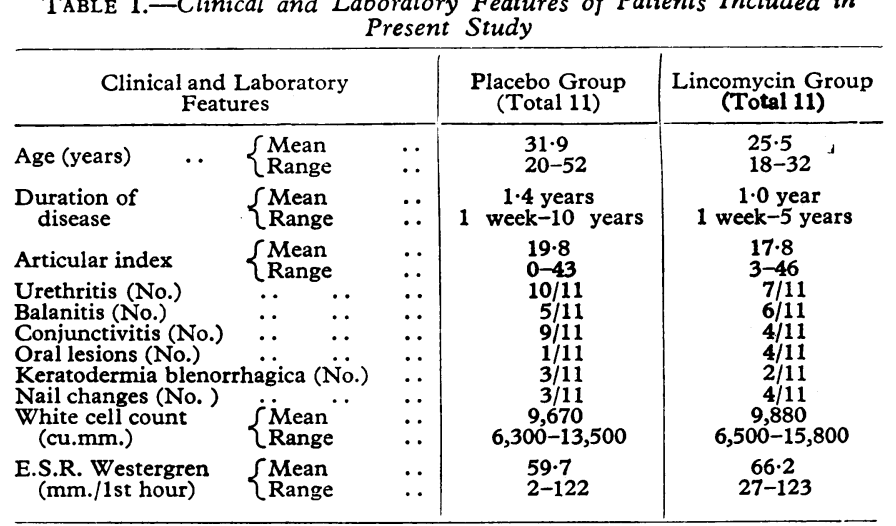

Throughout the four weeks of treatment no change was noted in the presence of urethritis, balanitis, conjunctivitis, and skin nail lesions. White cell counts were also unchanged at the end of the treatment period. The only changes observed were in the E.S.R. and articular index. Table II shows the fall in E.S.R. at weekly intervals compared with the starting level. Table III indicates the weekly fall in the articular index compared with the initial value. No difference was noted in the fall in E.S.R. or in articular index in both groups of subjects at any time during the four weeks of therapy.

TABLE II-Weekly Fall in Erythrocyte Sedimentation Rate in Patients with Reiter's Disease (All Values Related to Week 0)

\begin{tabular}{|c|c|c|c|c|}
\hline & $0-1$ & $0-2$ & $0-3$ & $0-4$ \\
\hline Lincomycin $\left\{\begin{array}{l}\text { Mean fall } \\
\pm \text { S.E. }\end{array}\right.$ & $\begin{array}{c}9.20 \\
+1.003\end{array}$ & $\begin{array}{r}7.457 \\
\pm 1.212\end{array}$ & $\begin{array}{r}6.300 \\
\pm 1 \cdot 362\end{array}$ & $\begin{array}{r}6.950 \\
+1.586\end{array}$ \\
\hline Placebo $\left\{\begin{array}{l}\text { Mean fall } \\
\pm \text { S.E. } \ldots\end{array}\right.$ & $\begin{aligned} & 9 \cdot 35 \\
\pm & 1 \cdot 441\end{aligned}$ & $\begin{array}{r}7 \cdot 986 \\
\pm 2 \cdot 139\end{array}$ & $\begin{array}{r}6.429 \\
\pm 1.623\end{array}$ & $\begin{array}{r}5 \cdot 300 \\
\pm 1 \cdot 125\end{array}$ \\
\hline
\end{tabular}

TABLE III.-Weekly Fall in Articular Index in Patients with Reiter's Disease (All Values Related to Week 0)

\begin{tabular}{l}
\hline \\
\hline
\end{tabular}

\section{Discussion}

This study was undertaken to evaluate the effectiveness of lincomycin hydrochloride in the treatment of Reiter's disease. During the period of therapy those subjects treated with the 
active preparation showed no significant improvement in clinical or laboratory findings, compared with a matched group of patients treated with placebo.

We have concluded that lincomycin hydrochloride is of no obvious benefit in the treatment of Reiter's disease.

This study was supported by a grant from the Arthritis and Rheumatism Council for Research in the United Kingdom. Lincomycin hydrochloride and placebo tablets were provided by Dr. J.
Warwick Buckler, of Boots Pure Drug Company. One of us (G. N.) was in receipt of a C.I.B.A. clinical research fellowship. Our thanks are due to Dr. W. Watson Buchanan for helpful criticism.

\section{REFERENCES}

Mowat, A. G., Chalmers, T. M., Alexander, W. R. M., and Duthie, J. R (1967) British Medical fourmal, $1,478$. Rinkoff, S. (1952). Fournal of the American Medical Association, 148 740

Ritchie, D. M., et al. (1968). Quarterly fournal of Medicine, 37, 393.

\title{
Effect of Tetrabenazine on Extrapyramidal Movement Disorders
}

\author{
M. A. DALBY,* M.D.
}

\begin{abstract}
Summary : Thirty patients with various extrapyramidal $\checkmark$ movement disorders were treated for prolonged periods with 75 to $225 \mathrm{mg}$. daily of tetrabenazine. In patients with choreiform and hemiballistic motor activity the involuntary movements were diminished or abolished. In patients with cerebellar or Parkinsonian tremor the tremor was aggravated in moderately severe cases, but was uninfluenced in severe cases. In all cases the dyskinesia returned when the drug was stopped.

Side-effects were inconsiderable and disappeared on reducing the dose slightly. Hence the drug may be an important alternative to neurosurgical treatment of hyperkinesia and especially suitable for severely disabled patients.
\end{abstract}

\section{Introduction}

Drug treatment of hyperkinetic syndromes aimed at the selective elimination of the abnormal motor movements began with the use of the drug reserpine for the treatment of Huntington's chorea (Farbrot, 1956 ; Forrest, 1957 ; Grung and Sundby, 1957 ; Brandrup, 1960). Nevertheless, even if a sufficiently high dose of the drug can reduce the choreiform activity in nearly all cases, this drug is not suitable for long-term treatment, as side-effects frequently occur in the form of hypotension, depression, and gain in body weight. The search for a less troublesome and more effective drug led to the introduction of tetrabenazine (Nitoman) (Brossi et al., 1958), for Huntington's chorea, hemiballismus, torsion dystonia, and the facial dyskinesias following phenothiazine medication (Brandrup, 1960 ; Møller-Christensen and Videbech, 1963 ; Sattes and Hase, 1964 ; Pakkenberg, 1968).

Tetrabenazine, like reserpine, is a monoamine-depletor and its pharmacological effect is, in principle, similar to that of reserpine. Clinical effects, however, start more quickly and the drug is eliminated in 24 to 48 hours. It is therefore easier to control the dose than using reserpine (Pletscher et al., 1962). The dose given has ranged from 75 to $300 \mathrm{mg}$. daily. Apart from slight sedation, which disappears on reducing the dose, no side-effects have been encountered in the cases of hyperkinesia treated.

- Senior Registrar, University Neurological Clinic, Aarhus Municipal Hospital, Denmark.

\section{Method}

Since 196230 patients with extrapyramidal movement disorders have been treated in a trial of the efficacy of tetrabenazine. Fourteen had choreiform movements, four had hemiballismus, two had dystonic torsion syndromes, four had intention tremor, and six had Parkinsonian tremor. The diagnostic classification was as follows: Huntington's chorea (8), cerebral arteriosclerosis (8), epidemic encephalitis (3), Sydenham's chorea (1), paralysis agitans (6), and spinocerebellar degeneration (4).

The results of treatment were evaluated independently by the nursing staff, by the patient's relatives, by the patient himself, and by me. In most cases treatment was given over several periods for one to four weeks alternating with periods of treatment with phenobarbitone or anti-Parkinsonian drugs. Thus the patient acted as his own control, but a placebo technique or a double-blind trial was not performed. In cases where an obvious effect was obtained treatment was extended for periods of three to six months. Two patients were treated for a period of six years without loss of effect.

Films taken of the patients before and during a period of treatment enabled close analysis of the character of the movements initially and of the change occurring during treatment. After discharge from hospital patients who had obtained relief from their hyperkinetic movements were given a choice of tetrabenazine, phenobarbitone, or anti-Parkinsonian drugs. In all cases they chose tetrabenazine. Routine blood tests, urine analysis, and liver function tests carried out during treatment showed no abnormalities.

\section{Results}

The Table shows the results of treatment with tetrabenazine in relation to the type of dyskinesia but without regard to the diagnostic classification. "Moderate" means a reduction of abnormal movements by $80-90 \%$ as judged by filming the patients. Cases classified as "good" showed complete cessation of the abnormal movements.

In patients with choreiform movements, whatever the aetiology, tetrabenazine produced a gradual decrease in the number and amplitude of the involuntary movements, first seen in the axial muscles of the trunk, then in the proximal muscles of the limbs, and lastly in the hands, feet, and face. These results 